\title{
Increasing the Templating Effect on a Bulk Insulator Surface: From a Kinetically Trapped to a Thermodynamically More Stable Structure
}

\author{
Chiara Paris, ${ }^{* \dagger}$ Andrea Floris, ${ }^{* \dagger,}$ Simon Aeschlimann, ${ }^{\S}$ Markus Kittelmann, ${ }^{\S}$ Felix Kling, ${ }^{\S}$ \\ Ralf Bechstein, ${ }^{\S}$ Angelika Kühnle, ${ }^{*}$, and Lev Kantorovich* ${ }^{\dagger} \dagger$ \\ ${ }^{\dagger}$ Department of Physics, King's College London, London WC2R 2LS, United Kingdom \\ ${ }^{\ddagger}$ School of Mathematics and Physics, University of Lincoln, Brayford Pool, Lincoln LN6 7TS, United Kingdom \\ ${ }^{\S}$ Institute of Physical Chemistry, Johannes Gutenberg University Mainz, Duesbergweg 10-14, 55099 Mainz, Germany
}

\section{Supporting Information}

\begin{abstract}
Molecular self-assembly, governed by the subtle balance between intermolecular and molecule-surface interactions, is generally associated with the thermodynamic ground state, while the competition between kinetics and thermodynamics during its formation is often neglected. Here, we present a simple model system of a benzoic acid derivative on a bulk insulator surface. Combining high-resolution noncontact atomic force microscopy experiments and density functional theory, we characterize the structure and the thermodynamic stability of a set of temperature-dependent

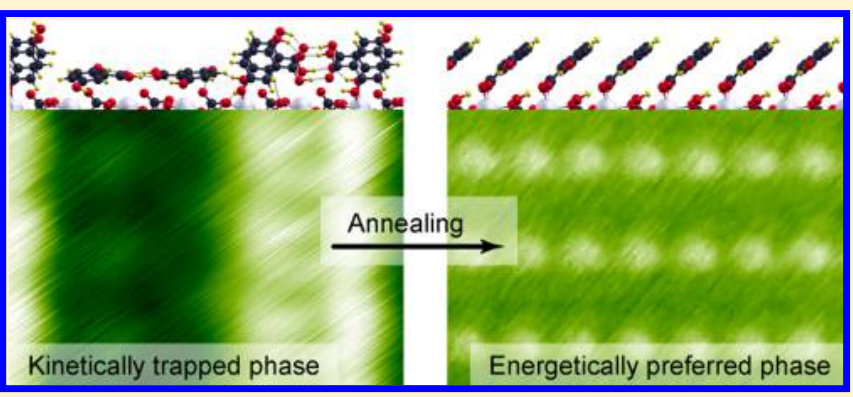
molecular phases formed by 2,5-dihydroxybenzoic acid molecules, self-assembled on the insulating calcite (10.4) surface. We demonstrate that a striped phase forms before the thermodynamically favored dense phase, indicating a kinetically trapped state. Our theoretical analysis elucidates that this stripedto-dense phase transition is associated with a distinct change in the chemical interactions involved in the two phases. The striped phase is characterized by a balance between molecule-molecule and molecule-substrate interactions, reminiscent of the molecular bulk. In contrast, the dense phase is formed by upright standing molecules that strongly anchor to the surface with a comparatively little influence of the intermolecular interactions, i.e., in the latter case the substrate acts as a template for the molecular structure. The kinetic trapping stems from a relatively strong intermolecular interaction between molecules in the striped phase that need to be broken before the substrate-templated dense phase can be formed. Thus, our results provide molecular level insights into two qualitatively different bonding motifs of a simple organic molecule on a bulk insulator surface. This understanding is mandatory for obtaining predictive power in the rational design of molecular structures on insulating surfaces.
\end{abstract}

\section{INTRODUCTION}

Molecular self-assembly is a well-established and powerful strategy for creating highly organized structures on surfaces with a well-defined functionality. ${ }^{1,2}$ Great achievements have been gained in this field ${ }^{2-5}$ especially on metallic substrates, mostly exploited due to their high reactivity with respect to adsorbed molecules and thanks to a large number of surface functionalization possibilities, resulting in metals work function shifts, charge transfer processes, long-range chiral structures, porous networks, anomalous coarsening, and so on. ${ }^{2-10}$

Although in many applications on molecular nanodevices, in molecular optics, ${ }^{11}$ electronics, ${ }^{12,13}$ and catalysis ${ }^{14}$ insulating substrates would be of great interest, achieving a comparable degree of structural control at the atomistic level remains a challenge for such systems. These systems are typically characterized by weak molecule-surface interactions as insulating materials are in general chemically poorly reactive. ${ }^{15-17}$ As a result, molecules are often observed to interact stronger with each other rather than with the substrate, ${ }^{18,19}$ forming less stable assemblies or bulk-like crystallites instead of monolayers, ${ }^{18,19}$ which constitutes a strong limitation for exploring self-assembly principles. For this reason, the study of molecular self-assembly has only recently been extended to insulators and versatile strategies to realize functionalized surfaces still need to be explored. ${ }^{20}$

However, insulating substrates characterized by highly localized charges, like ionic crystals, have shown to exhibit an increased binding to polar or charged molecules that can be anchored via electrostatic interaction to the surface ions. ${ }^{20}$ An important example in this respect is the calcite (10.4) surface, which owes its reactivity to the presence of a network of calcium ions and negatively charged carbonate groups. ${ }^{17}$ This has recently opened several possibilities to realize assembled structures of organic molecules on this insulating sub-

Received: May 29, 2016

Revised: July 20, 2016

Published: July 20, 2016 
strate. $^{20-23}$ One way of creating a stable wetting layer on the calcite (10.4) surface has been proposed via the deprotonation of a benzoic acid derivatives. ${ }^{24,25}$ In the latter study, noncontact atomic force microscopy (NC-AFM) experiments performed at room temperature ${ }^{25}$ have revealed that the 2,5-dihydroxybenzoic acid (DHBA) molecules form two ordered phases: one consisting of alternating stripes with different apparent height (a striped phase) and the other, more uniform, made of a network of regular spots (a dense phase). The striped phase has been observed to completely disappear over time at room temperature while, correspondingly, existing dense phase islands have increased in size and number. On the grounds of Kelvin probe force microscopy measurements, the stable dense phase has tentatively been assigned to be the result of a deprotonation reaction. ${ }^{25}$ These results provide an indication that the observed anchoring mechanism might be of more general nature. Thus, this mechanism might serve as a promising strategy for molecular anchoring, given that the underlying physical principles are understood.

In this work, we perform a detailed study of the structure and thermodynamics of several phases formed by DHBA molecules upon deposition on a calcite (10.4) surface. First, we present the results of NC-AFM experiments performed in a wide temperature interval, revealing a sequence of irreversible phase transitions and the appearance of additional phases made of molecular clusters. Next, we analyze in detail the structure and stability of the striped and dense phases by characterizing their atomistic structure and highlighting their drastically different chemical bonding with the substrate.

\section{RESULTS}

Using NC-AFM high-resolution experiments, we imaged DHBA molecules on the calcite (10.4) surface (Figure 1) at

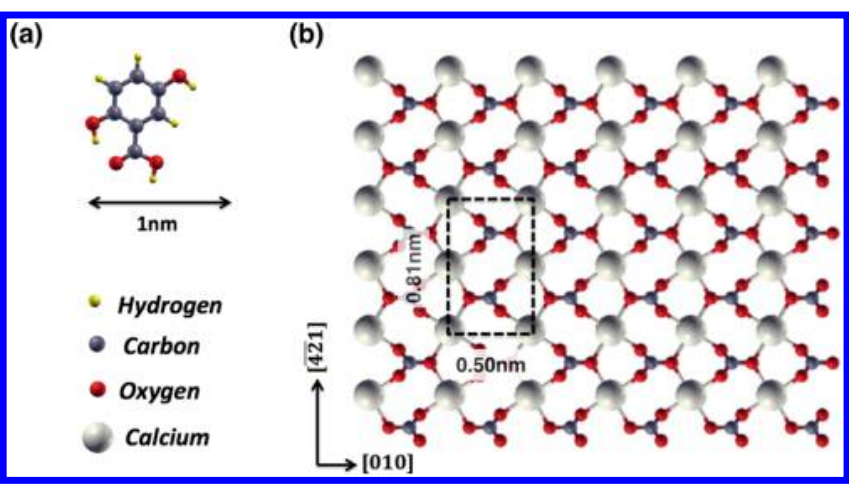

Figure 1. DFT relaxed structures of (a) a DHBA molecule in the gas phase and (b) the calcite (10.4) surface (top view of the top layer). The rectangular unit cell (shown by the dashed line) consists of two calcium ions and two carbonate groups rotated by $60^{\circ}$ with respect to each other.

temperatures ranging from about 100 to $400 \mathrm{~K}$ (Figure 2). The images were obtained at the indicated sample temperature after depositing 0.4 monolayers of molecules onto a substrate held at about $100 \mathrm{~K}$ (see Supporting Information for details). The images allow identifying of at least four different phases formed by DHBA on calcite (10.4), each existing within a certain temperature range (Table 1 and Figure 2), with one molecular phase transforming into the next within a specific temperature interval, where two phases coexist. The four phases exhibit

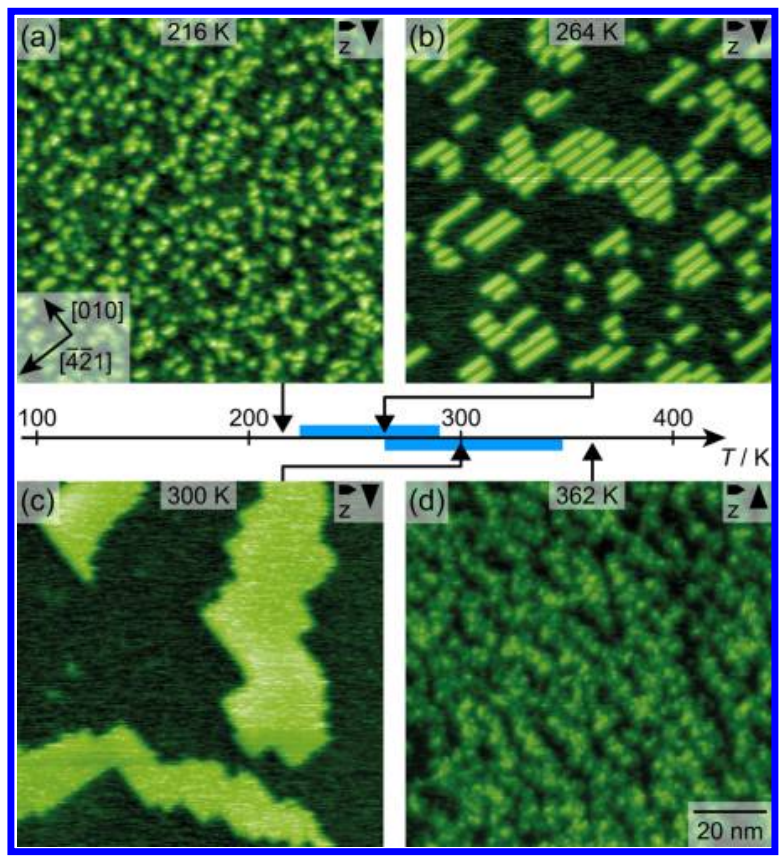

Figure 2. Overview of various molecular arrangements (four phases) as a function of the sample temperature. The blue color on the temperature scale indicates the temperature ranges in which the striped phase (from 224 to $290 \mathrm{~K}$ ) and the dense phase (from 264 to $348 \mathrm{~K}$ ) are observed in the experiment. (a) Clusters; (b) well-ordered one-dimensional striped islands; (c) well-ordered two-dimensional dense islands; (d) clusters. A plane subtraction was applied to the data.

Table 1. Summary of the Observed Molecular Structures on the Calcite (10.4) Surface after Deposition of DHBA and Heating to the Indicated Sample Temperature

\begin{tabular}{lll}
\multicolumn{1}{c}{ structure } & temp $(\mathrm{K})$ & \multicolumn{1}{c}{ description } \\
phase 1 "clusters" & $109-216$ & tightly packed clusters \\
phase 1 and 2 & $224-241$ & clusters and stripes \\
phase 2 "striped" & $244-264$ & striped islands \\
phase 2 and 3 & $264-290$ & striped and dense islands \\
phase 3 "dense" & $291-348$ & dense islands \\
phase 4 "clusters" & $361-406$ & dissolved islands \\
\hline
\end{tabular}

completely different structural features, as we will describe in the following.

At low temperature, we observe only molecular clusters, which are homogeneously distributed on the surface (phase 1, Figure 2a). Upon annealing the sample to $224 \mathrm{~K}$ we start to observe the formation of ordered molecular stripes (phase 2, Figure $2 \mathrm{~b}$ ), which coexist with the cluster phase up to $241 \mathrm{~K}$. At $244 \mathrm{~K}$ all clusters have disappeared, and only individual stripes are found on the surface. At even higher temperature, the stripes rearrange to form islands consisting of stripes, an assembly that was denoted as striped phase. ${ }^{25}$ It has been suggested that the stripes consist of a bulk-like structure, forming a $(6 \times 1)$ superstructure with a unit cell of $3.0 \mathrm{~nm} \times$ $0.8 \mathrm{~nm}$ along [010] and $[\overline{42} 1]$ directions, respectively ${ }^{25}$ (Figure 3a).

At $264 \mathrm{~K}$ we start to observe a phase transition to a completely different assembly, formed by well-ordered twodimensional dense islands (phase 3, introduced as dense phase, Figure 2c). At room temperature (291 K), the striped phase is observed to vanish completely, and only dense phase islands are observed after some hours, in line with an earlier work. ${ }^{25}$ 


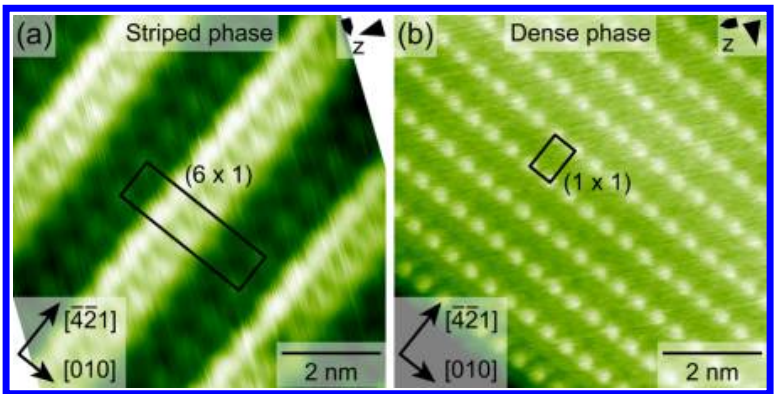

Figure 3. Close view of drift-corrected NC-AFM high-resolution images of striped and dense phase. (a) The striped island (phase 2) consists of a pair of double rows running along the [ $\overline{42} 1]$ direction. The $(6 \times 1)$ unit cell is marked by a rectangle. (b) An image of a dense island (phase 3$)$, exhibiting a $(1 \times 1)$ structure.

Originally, the dense phase was assigned to a $(1 \times 5)$ superstructure with a rectangular unit cell containing six molecules $(0.5 \mathrm{~nm} \times 4.1 \mathrm{~nm})$. However, further AFM experiments performed in this work allow us to establish a slightly less dense $(1 \times 1)$ structure containing one molecule per calcite unit cell (Figure $3 b$ ), as also confirmed by our theoretical analysis (see below). Finally, after annealing to 361 $\mathrm{K}$, the dense islands have dissolved again and small clusters, distributed homogeneously on the surface (phase 4, Figure 2d) are observed.

Importantly, we observed that all three phase transitions are irreversible when cooling the sample again. In particular, the striped phase does not reappear at $264 \mathrm{~K}$ on a substrate annealed before to $300 \mathrm{~K}$, where only the dense phase had formed. In an experiment with a sample covered by striped and dense phase islands, the observed phases remained unchanged upon cooling to $215 \mathrm{~K}$. These experiments indicate that the dense phase must be thermodynamically more favorable than the striped phase within a wide range of temperature, i.e. even when the striped phase is observed in the experiment, suggesting that the latter is kinetically trapped (see discussion at the end of the section).

In the following, we discuss theoretical simulations of the striped and dense phases to elucidate their structures and specific binding configurations, examining also the essential points necessary to understand their different stability. We first focus on the striped phase by studying the energetics of a number of configurations compatible with the experimental (6 $\times 1$ ) unit cell. The NC-AFM image (Figure 3a and ref 24) shows a sequence of alternating bright and dark stripes running along the $[\overline{42} 1]$ direction. Hence, we first considered structures where two sets of differently oriented molecules are present on the surface: one set lying flat on calcite, compatible with the dark stripes when periodically repeated, and the other lying nearly perpendicular to the surface, compatible with the bright stripes.

These configurations are reminiscent of structures found in the bulk crystal formed by DHBA molecules. Here DHBA molecules form planar ribbons of dimers connected via intermolecular hydrogen bonds between the carboxylic groups. ${ }^{26}$ More in detail, inside the bulk structure we identified two cutting planes consisting of six molecules per cell (see Figure 4). Both units were used as a first guess for the unit cell of the striped phase. In the first configuration (Figure 4a), two dimers $[\mathrm{A}]$ are nearly parallel to each other and interact via $\mathrm{H}$ bonds with a further dimer [B], which lays almost perpendicular to the dimers $[\mathrm{A}]$. The second configuration
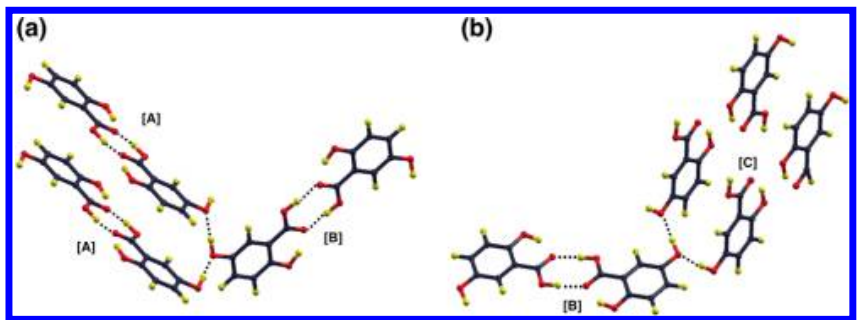

Figure 4. Two cutting planes of the DHBA bulk crystal. (a) Six molecules are arranged in three pairs of dimers: two almost parallel to each other $([\mathrm{A}])$ and one perpendicular to them $([\mathrm{B}])$. $[\mathrm{A}]$ dimers interact with each other via $\mathrm{H}$-bonds between the carboxylic groups and with $[\mathrm{B}]$ via $\mathrm{H}$-bonds between the hydroxyl substituents of the benzene rings. (b) In this configuration, we have one dimer lying flat ([B]) and four upright molecules not forming a dimeric structure. Intermolecular hydrogen bonds are shown by dashed black lines.

(Figure $4 \mathrm{~b}$ ) is rather similar to the first, but the parallel molecules [C] do not form dimers. When optimizing both configurations on top of the $(6 \times 1)$ calcite cell, we find the second being by $1 \mathrm{eV}$ per cell higher in energy with respect to the first. For a better and exhaustive analysis of the stability, we considered also structures with four and eight molecules, not necessarily inheriting their configuration from the molecular bulk or being compatible with the observed stripes (see the Supporting Information for details) but still sterically fitting the $(6 \times 1)$ calcite cell. Overall, the most stable structure found is the one with six molecules per cell as shown in Figure 5. It is made by three pairs of dimers, one lying flat and two almost vertically on top of the substrate. Importantly, this configuration, completely organized in dimers, is the only one closely resembling the bulk ribbon motif shown in Figure 4a, suggesting that the dimeric arrangement is a key ingredient for the stabilization of the striped phase.

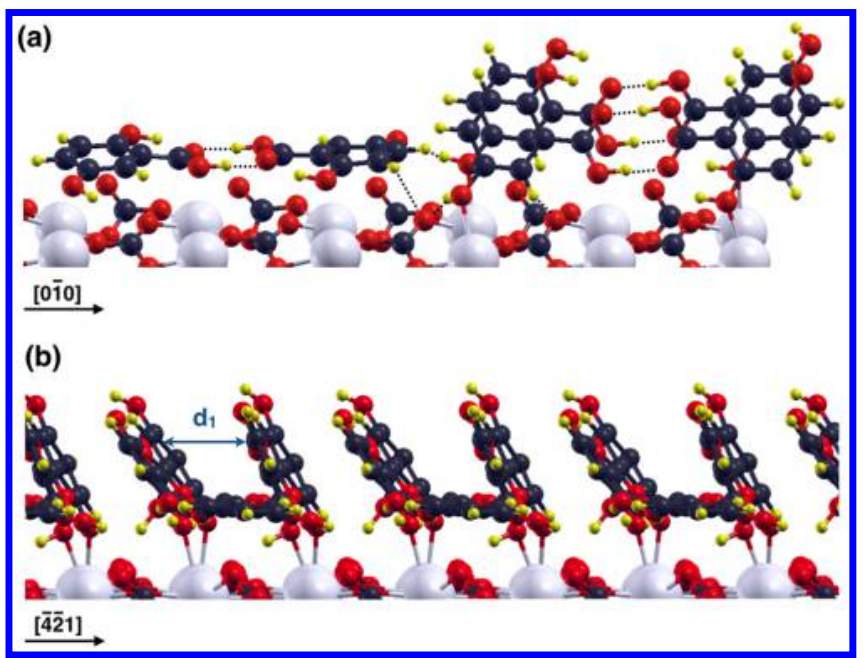

Figure 5. DFT calculated striped phase structure. (a) A view along the [010] direction, which highlights the intra- and interdimer hydrogen bonds. The flat dimers interact with the calcite surface via van der Waals interaction and H-bonds between the hydroxyl groups of the molecules and the oxygen atoms of the surface carbonate groups. (b) A view along the $[\overline{42} 1]$ direction, showing the tilting of the upright dimers to maximize their $\pi-\pi$ interaction, where the optimal distance between the centers of two benzene rings is $d_{1}=4.3 \AA$. The upright dimers interact with the substrate mainly via electrostatic bonds between the negatively charged oxygen atoms of the hydroxyl substituents and the surface calcium ions. 
By partitioning the striped phase structure (Figure 5) in various subsystems (always kept fixed in the striped phase geometry), such as pairs of individual dimers and different dimers and the surface, we estimated semiquantitatively the interaction energies between the dimers and those with the surface (the details of the calculations are given in the Computational Methods section and Supporting Information).

The relative contribution from each type of bonding is illustrated in Figure 6. Upright dimers interact with each other

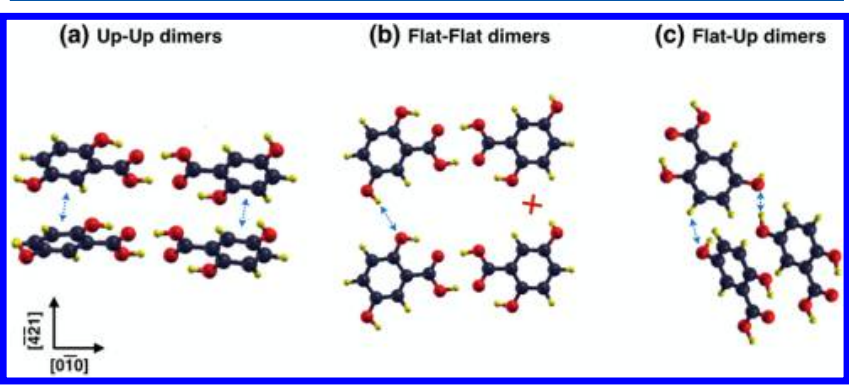

Figure 6. Interdimer bonding within the striped phase adlayer. (a) Upright dimers interact via $\pi$-stacking between the benzene rings (27\% of the total interaction energy). (b) Flat dimers along the [ $\overline{42} 1]$ direction interact via $\mathrm{H}$-bonds between the hydroxyl groups of the benzene rings, marked in the picture with blue arrows ( $11 \%$ of the total interaction energy). Note that the hydroxyl groups marked with a red cross in between form H-bonds with the surface instead. (c) Flat and upright dimers (within the same cell) interact weakly via H-bonds between the hydroxyl groups (5.5\%). The rest of the interaction energy $(\sim 56 \%)$ is provided by the adlayer-surface bonding.

via $\pi$-stacking (Figure 6a) while flat dimers via H-bonds (Figure $6 \mathrm{~b})$. The interaction between neighboring flat and upright dimers is relatively weak and is due to hydrogen bonding (Figure 6c). The molecule-substrate interaction is provided by the upright dimers mainly via electrostatic interactions with the surface (Figure $5 \mathrm{~b}$ ) and by the flat dimers via $\mathrm{H}$-bonds and van der Waals interactions (Figures $5 \mathrm{a}$ and $6 \mathrm{~b}$ ). Hence, in the striped phase the molecule-molecule and the moleculesubstrate interactions are at balance, accounting for $\sim 44 \%$ and $\sim 56 \%$ of the interaction energy of the whole phase, respectively. The large contribution of the molecule-molecule interaction is consistent with the fact that this phase (unlike the dense phase described below) is reminiscent of the DHBA bulk, where only dimers are present.

The theoretically obtained unit cell dimensions $(3.03 \mathrm{~nm} \times$ $0.81 \mathrm{~nm})$ are in excellent agreement with the experimental values. The obtained theoretical structure is in good qualitative agreement with the NC-AFM images, where brighter spots are assigned to the upright dimers and darker areas to the flat structures (see Figure 7).

In contrast to the striped phase, the dense phase appears as a uniform molecular organization on calcite (10.4), as shown by the high-resolution NC-AFM image in Figure 3b. Previous experiments $^{25}$ done at room temperature suggested that the phase is formed by upright molecules tentatively assigned to deprotonated molecules, a structure clearly no longer based on dimers. Hence, before presenting the theoretical results on the dense phase structure, we first analyze the adsorption geometry of a single DHBA molecule on the surface. The most stable structure found corresponds to a flat-lying molecule (see Figure 8a) with the oxygen atoms of the carboxylic group being close to two calcium atoms, indicating an electrostatic interaction.

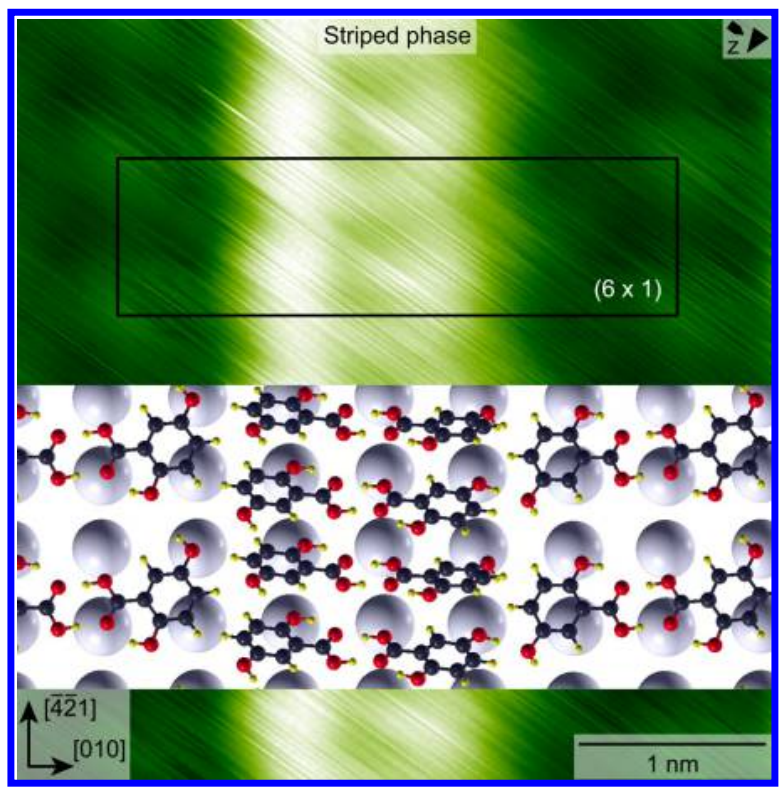

Figure 7. High-resolution NC-AFM image of the striped phase with a top view of the calculated adlayer (for clarity, in the superimposed atomic model only the topmost surface calcium atoms are shown), highlighting the alternating rows of flat and upright molecules.
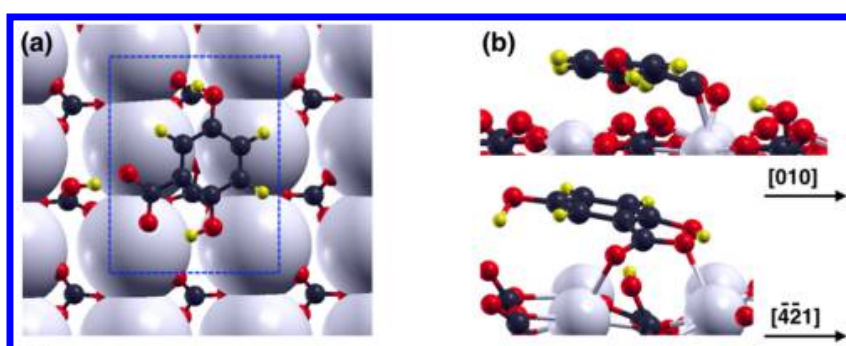

(c)
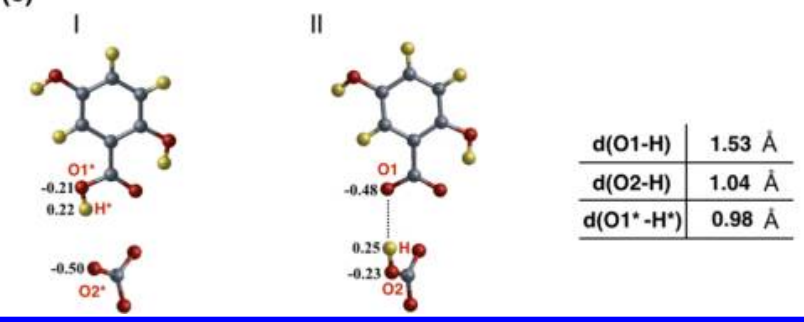

Figure 8. DFT relaxed geometry of a single deprotonated molecule on the surface. (a) Top view of the structure showing that the single molecule occupies almost an entire calcite unit cell (highlighted by a dotted blue rectangle). Note that the size of the calcium ions was deliberately increased for a better visualization. (b) A view of the

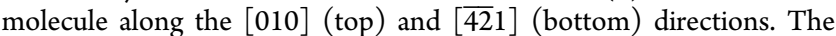
single molecule is almost flat on top of the surface with the oxygen atoms of the carboxylic group being close to two calcium atoms. Furthermore, the hydrogen atom of the carboxylic group is close to the oxygen of the nearest carbonate group. (c) Relevant Mulliken charges of the atoms in the isolated molecule and the clean surface $\left(\mathrm{Ol}^{*}, \mathrm{H}^{*}\right.$, and $\mathrm{O}_{2} *$ in I) are compared to the ones of the single adsorbed molecule $(\mathrm{O} 1, \mathrm{H}$, and $\mathrm{O} 2$ in $\mathrm{II})$. The oxygen-hydrogen distances $\left(d\left(\mathrm{O} 1^{*}-\mathrm{H}^{*}\right), d(\mathrm{O} 1-\mathrm{H})\right.$, and $\left.d(\mathrm{O} 2-\mathrm{H})\right)$ are provided for both cases.

Furthermore, the hydrogen atom of the carboxylic group is close to the oxygen of the nearest carbonate group (see Figure $8 b$ ). The Mulliken charge analysis ${ }^{27}$ (see Supporting Information) reveals that this hydrogen atom has a charge of $+0.22 e$ in the isolated molecule (Figure $8 \mathrm{c}, \mathrm{I}$ ) and of $+0.25 e$ 
when the molecule is adsorbed on the surface (Figure 8c, II). The charge of the oxygen atom of the surface is $-0.50 \mathrm{e}$ for the clean surface $(\mathrm{O} 2 *)$ and changes to $-0.23 e$ when the molecule adsorbs to the surface $(\mathrm{O} 2)$. At the same time, the charge of the oxygen atom of the molecule changes from $-0.21 e$ of the isolated molecule $\left(\mathrm{O} 1^{*}\right)$ to $-0.48 e$ for the adsorbed molecule (O1). Therefore, there is charge transfer of $-0.27 e$ from the surface oxygen atom $(\mathrm{O} 2)$ to the oxygen atom of the molecule (O1). The available KPFM data ${ }^{25,28}$ are in line with the interpretation on the charge transfer made here. Moreover, from Figure $8 \mathrm{c}$, we see that the $\mathrm{O}-\mathrm{H}$ distance $d(\mathrm{O} 2-\mathrm{H})=$ $1.03 \AA$ between the $\mathrm{H}$ atom adsorbed on the surface and the surface $\mathrm{O}$ atom is similar to the $\mathrm{O}-\mathrm{H}$ distance $d(\mathrm{O} 1 *-\mathrm{H})=$ $0.96 \AA$ in an isolated molecule. The distance between the oxygen atoms $d(\mathrm{O} 1-\mathrm{O} 2)=2.57 \AA$ is comparable with the oxygen-oxygen distance in a typical hydrogen bond. The charge and distance analysis thus suggests that the oxygen atoms of the molecule and the surface switch role upon adsorption. On the basis of our results, we see the distance between hydrogen and its "parent" oxygen in the molecule increases upon adsorption, explaining the tentative assignment to a deprotonation. The interaction between the oxygen of the molecule and the newly formed surface $\mathrm{OH}$ group can be ascribed to a hydrogen bond. Further stability is given by the van der Waals interaction between the benzene ring of the molecule and the surface. We also find that taking the hydrogen atom further away from the "parent" molecule is energetically less favorable.

Our single molecule analysis guided us in constructing various models for the dense phase, which are compatible with the experimentally observed structure. We first considered a monolayer with one upright molecule in each calcite cell (fitting a flat molecule in the same cell is sterically unfeasible) as suggested by our current NC-AFM images. Since the AFM images do not disclose the specific binding configuration of all molecules, a $(2 \times 5)$ cell with ten molecules was considered. This enabled us to vary the number of molecules with increased hydrogen-oxygen distance (as in the case of a single adsorbed molecule), ranging from none to all molecules having their hydrogen atoms placed at an increased distance (see the Supporting Information for details).

The most energetically favorable structure is shown in Figure 9 and its comparison with the NC-AFM image in Figure 10. In this structure, all molecules are equivalent (they have a $(1 \times 1)$ arrangement, with one molecule per calcite unit cell), upright standing, and with an increased hydrogen-oxygen distance as in the case of a single adsorbed molecule. This situation can be described by two electrostatic bonds of the oxygen atoms of the carboxylic group with the surface calcium atom as well as an $\mathrm{H}$ bond of the carboxylic group of the molecule with the carbonate group of the surface. As shown in Figure 9a, the molecules do not stand strictly upright: they adsorb with a rather large tilt angle $\left(\alpha \sim 39^{\circ}\right)$, which optimizes the $\pi-\pi$ interaction between DHBA molecules belonging to adjacent rows along [010] (the center-center benzene rings distance is $\sim 3.5 \AA$ ). Moreover, the stacking of the molecules with the benzene rings parallel to [ $\overline{42} 1]$ also favors the $\mathrm{H}$-bond between the benzene rings hydroxyl substituents of adjacent molecules, with an optimized oxygen-oxygen distance of $2.30 \AA$ (see Figure 9b).

For the calculation of the relative energy contributions to the dense phase interaction energy we followed two different approaches (see Supporting Information), which, despite

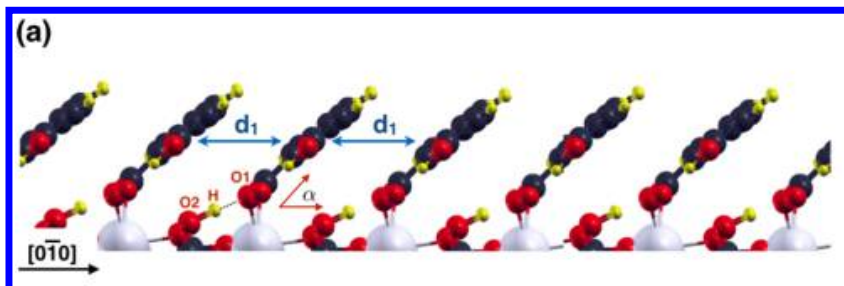

(b)

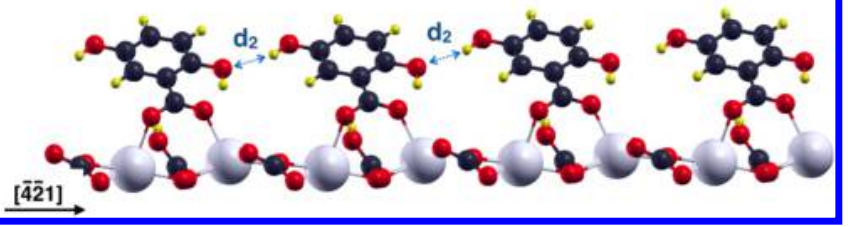

Figure 9. DFT calculated dense phase structure. (a) A view along [0ㅣ] direction, showing the $\pi-\pi$ distance $\left(d_{1}=3.5 \AA\right)$ between benzene rings, and the hydrogen atom binding to the nearest protruding surface oxygen. The $\mathrm{O} 2-\mathrm{H}$ distance is $1.04 \AA$ while $\mathrm{O} 1-\mathrm{H}$ is $1.41 \AA$. The tilt angle $\alpha$ of the molecule with respect to the surface plane is about $39^{\circ}$. (b) A view along the $[\overline{42} 1]$ direction. The oxygenhydrogen distance between adjacent molecules $d_{2}=2.30 \AA$.

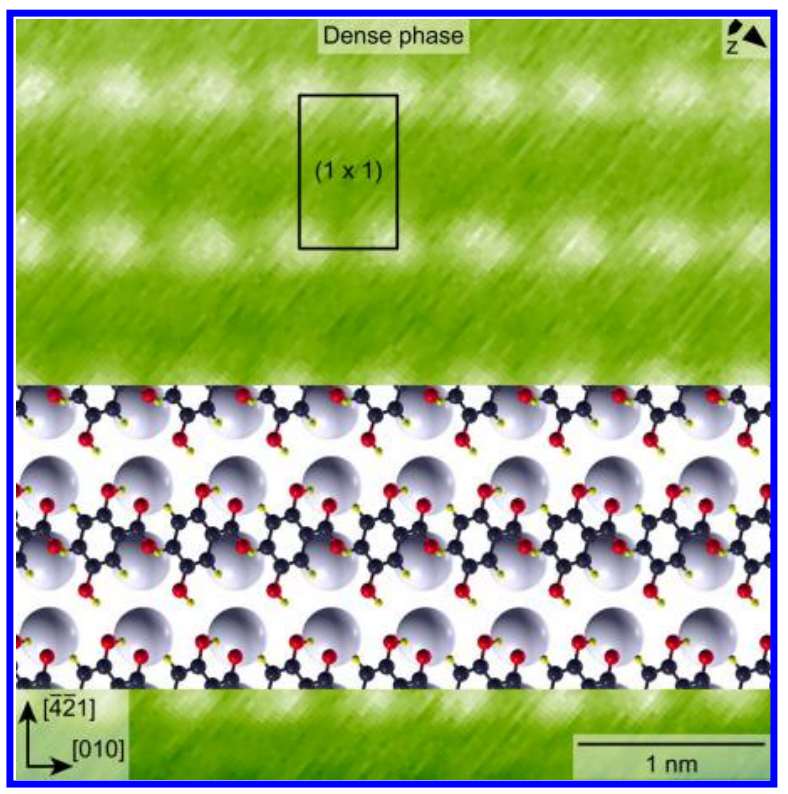

Figure 10. Calculated dense phase atomic model is superimposed on the experimental NC-AFM image from Figure 3b. For clarity, in the atomic model only the topmost surface calcium atoms are shown.

yielding slightly different numerical results, do not affect our main conclusions. The derived picture differs substantially from the one of the striped phase, as the major contribution to the total interaction energy of the dense phase originates from the molecule-surface interaction. Here, only between $8 \%$ and $14 \%$ is due to the molecule-molecule interaction, while between $86 \%$ and $92 \%$ of the contribution comes from the moleculesurface interaction (depending on the method used). More in

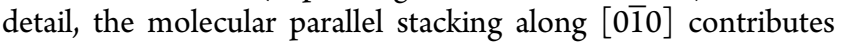
about $4 \%$, which is $0.10 \mathrm{eV}$ per molecule, an expected energy gain for aromatic-aromatic $\pi-\pi$ interaction $^{29}$ (Figure 9a). Importantly, three times as much comes from the lateral $\mathrm{H}$ bond between the molecules running along [ $\overline{42} 1]$ (Figure 9b). 
Therefore, the arrangement of the molecules within this phase is not only a collection of individually adsorbed molecules, as there is a significant intermolecular interaction, which affects the actual geometry of the molecules in the layer. In fact, an isolated upright standing molecule adsorbed on the surface is by $0.80 \mathrm{eV}$ higher in energy than the flat molecule adsorbed on the surface (Figure 8a,b). The collective effect due to the intermolecular interaction enables the molecules to stand at a larger angle to the surface, if compared to the single molecule case.

Importantly, a comparison of the free energies for the two phases reveals that the dense phase is thermodynamically more stable than the striped phase in the temperature window of $200-400 \mathrm{~K}$, i.e. even in the temperature range where we obtain the striped phase experimentally. The calculated DFT energy difference of the two phases is $0.65 \mathrm{eV}$ (relative to the common $(6 \times 2)$ cell), while the contribution of atomic vibrations to the free energy difference adds an additional $0.25-0.30 \mathrm{eV}$ (within the temperature range considered for the two phases), making the dense phase even more favorable (see the Supporting Information for more details).

We conclude our analysis with a discussion about the transition from the striped to the dense phase. Our experimental results (see Table 1) indicate an increased transition rate upon increasing the temperature. As a consequence, there must be a certain energy barrier for the system to undergo the phase transition from the striped to the dense phase. Note that as stated above, according to our DFT calculations the dense phase is more favorable than the striped one even within the temperature interval where the latter is observed experimentally and that the striped phase is not regained upon cooling the dense phase.

Why then does the striped phase appear at all? To address this question, it is instructive to compare the stability of a molecular dimer in the gas phase with that on the surface. In both cases the two molecules form a double H-bonding at the carboxylic group (see Figure 11). However, while in the gas phase our calculations indicate the dimer as $0.8 \mathrm{eV}$ more favorable than two single molecules, on the surface we have the opposite picture; i.e. the dimer is by $0.5 \mathrm{eV}$ less stable than two adsorbed individual molecules. Importantly, we have also found

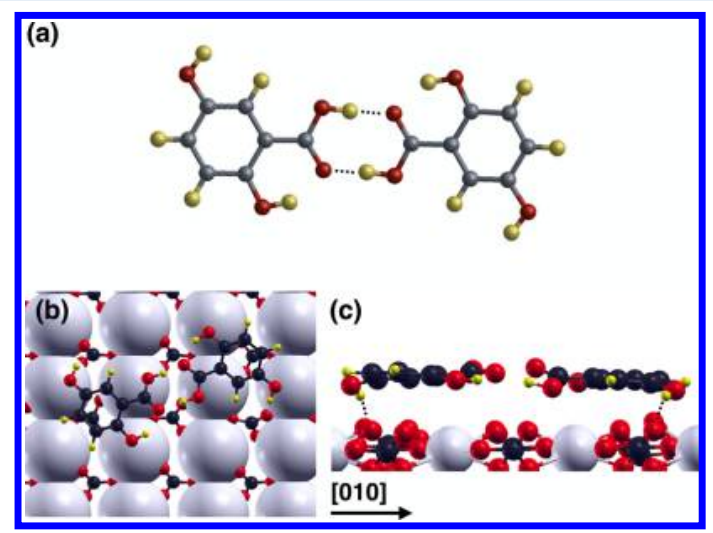

Figure 11. Calculated geometries of a molecular dimer in the gas phase (a) and on the calcite surface (b, c). In (b) the dimer lies flat on the surface with an orientation that maximizes the $\mathrm{H}$-bonding interactions between the lateral hydroxyl substituents on the benzene rings with the calcite oxygen atoms. (c) A view of the dimer along the [010] direction. The H-bond interactions between the dimer and the surface are marked with black dotted lines. that the energy barrier for a dimer dissociation process on the surface is rather large $(\sim 1.3 \mathrm{eV}$, see the Supporting Information for details). On the basis of these results, the following explanation can be put forward for the fact that the less favorable striped phase appears before the dense one at low enough temperatures. Since dimers are energetically favored to form in the gas phase, a considerable number of molecules would form dimers during evaporation, prior to their deposition on the surface. Hence, phase 1 will form first, where small clusters consisting preferentially of dimers are formed on the surface. Because of the mentioned large energy barrier necessary to dissociate the dimers, they remain stable within a considerable temperature interval. Hence, with appropriate temperature increase the diffusion of dimers would naturally result in them joining together in the striped phase. This must be the most preferable mechanism, as the formation of the dense phase directly from diffusing dimers at low temperatures would correspond to surmounting a much larger energy barrier associated with breaking of the relatively strong double $\mathrm{H}$-bond between the molecules. As the temperature increases, the dimer dissociation kicks in, leading to the appearance of many single molecules, which, due to the similarity of their adsorption geometry to the molecular structure in the dense phase, initiate the nucleation of the latter.

The above considerations indicate that there must be a smaller energy barrier for the formation of the striped phase compared to the barrier for the direct formation of the dense phase. Therefore, it is kinetically more favorable going first to a phase made out of dimers such as the striped phase, and only then to the dense phase via a phase transition, which must be thermally activated. Hence, the striped phase must be kinetically trapped. Thus, the fact that the striped phase appears prior to the dense phase indicates the importance of the specific initial molecular arrangement on the surface immediately after deposition that favors its formation.

\section{CONCLUSION}

In this work, we investigated the temperature evolution of the assembly formed by DHBA molecules on the calcite (10.4) insulating substrate by combining high-resolution NC-AFM experiments and state-of-the-art DFT calculations. The temperature increase triggers a series of irreversible transitions between several molecular phases indicating a drastic change of the interactions at play.

Our analysis is focused on two ordered phases, denoted as striped and dense. While the NC-AFM imaging of the first phase reveals a regular alternation of bright and dark stripes, for the second one a uniform and close-packed network of bright spots is observed. Over time, the striped phase transforms into the dense, which above $290 \mathrm{~K}$ remains the only phase present before dissolving into clusters at even higher temperature. A very detailed theoretical analysis was performed to identify the atomic arrangements of the two phases and the different stabilizing factors at play in each of them. The striped phase is made out of upright and flat-lying dimers and is reminiscent of the DHBA molecular bulk. The dense phase is thermodynamically more stable than the striped phase. It is formed by a network of upright monomers, strongly anchored to the surface both electrostatically and by an H-bond.

Our analysis of the contributions to the total interaction energy due to the molecule-molecule and molecule-surface interactions disclosed a significantly different binding configuration within the two phases. Compared to the balanced 
interactions in the striped phase, in the dense phase the lack of a dimeric molecule-molecule interaction is mediated by a much stronger interaction of the molecules with the surface, resulting in a strong substrate templating effect.

Importantly, our results show that using an annealing procedure it is possible to reach an assembly (the dense phase) on the calcite (10.4) surface, which is not directly accessible when the molecules are deposited at room temperature: upon deposition a self-assembled monolayer of the striped phase is created first, and only then, via a slow phase transition, a thermodynamically more stable dense phase structure is formed. We argue that the formation of the striped phase at lower temperature prior to the transformation to the dense phase is due to kinetic trapping, as strongly suggested by the irreversible character of the phase transition and by our free energy calculations that identify the dense phase as energetically more favorable within a wide temperature interval.

The discovered anchoring mechanism of the carboxylic group changing from intermolecular hydrogen bonding in the striped phase to a strong molecule-surface interaction in the dense phase is likely to be of general nature for the carboxylic acid moiety. Molecular side groups can be then suitably chosen to provide additional interaction between neighboring molecules. We believe that these factors will play an important role in designing stable molecular architectures on surfaces of insulating crystals such as calcite. Identifying specific driving forces, as this one, is essential in the direction of potential technological applications of self-assembled structures on insulators.

\section{COMPUTATIONAL METHODS}

Density functional theory (DFT) calculations were mainly performed with the Quickstep code ${ }^{30}$ within the CP2K package, $^{31}$ using a mixed Gaussian and plane waves basis set, the Goedecker, Teter and Hutter (GTH) pseudopotentials, ${ }^{32}$ and a GGA-PBE ${ }^{33}$ exchange-correlation functional including a Grimme-D2 van der Waals interaction. ${ }^{34}$ We used a plane-wave basis energy cutoff of $600 \mathrm{Ry}$ and the $\Gamma$ point to sample the Brillouin zone. In all calculations large enough cells were considered to justify the latter approximation. The calcite substrate was modeled with a periodically repeated slab of three layers, allowing a vacuum gap between the adsorbed adlayer and the bottom layer of the slab above it of $\sim 30 \AA$. Relaxations were considered completed when atomic forces were $<0.02 \mathrm{eV} /$ A. Only atoms belonging to the two uppermost top layers and all atoms of the molecules were allowed to relax. The most relevant energy comparisons were also performed using the plane-wave pseudopotential package Quantum-ESPRESSO. ${ }^{35}$ In order to analyze the stability of both phases, we performed single point energy calculations of the interaction energies between different elementary components of the systems (pairs of neighboring molecules, molecule, and the surface). The interaction energy is understood as a difference between the total energy of the whole system minus the sum of the total energies of all its components calculated in the geometry of the whole system. Pre- and postprocessing were done using the TETR/LEV00 package. ${ }^{36}$ In order to estimate the charge transfer between the adsorbed molecules and the calcite surface, one has to compare the charge densities before and after adsorption. Although different methods can be used to estimate this, quantitative analysis can only be done based on a somewhat artificial definition of what is meant by an atom in a many-atomic system. Since we are interested here in a qualitative analysis only, we have used the Mulliken population analysis for this purpose. Atomic vibrations were calculated using the frozen-phonon method with the free energy obtained within the quasi-harmonic approximation. ${ }^{37}$ Nudged elastic band $(\mathrm{NEB})^{38}$ calculations were performed using nine replicas, including the initial and final states. In these calculations all atoms of the molecules and only the surface atoms allowed to relax were displaced.

\section{EXPERIMENTAL METHODS}

All sample preparation and measurements were performed under ultrahigh vacuum (UHV) conditions with a base pressure $<10^{-10}$ mbar. Optical quality calcite $\left(\mathrm{CaCO}_{3}\right)$ crystals (Korth Kristalle $\mathrm{GmbH}$, Kiel, Germany) were cleaved in situ prior to the deposition of 2,5-dihydroxybenzoic acid (DHBA, Aldrich, Munich, Germany). Heating the DHBA molecules to $67^{\circ} \mathrm{C}$ results in sublimation and adsorption onto the calcite substrate, which was precooled with liquid nitrogen to about $100 \mathrm{~K}$; additional details can be found in ref 24 . The temperature of the sample was regulated via a liquid nitrogen continuous flow cryostat with integrated counter heating element. A copper braid connects the heat exchanger and the sample holder stage. The temperature stability at the heat exchanger, measured with a silicon diode, is better than $0.1 \mathrm{~K}$ during AFM experiments. Sample temperatures stated in this article were measured at the sample holder stage using a Pt-100 sensor with an accuracy of $\pm 1 \mathrm{~K}$. Note there is an unknown temperature difference between sample surface and sample holder stage, which is estimated by the manufacturer to be less than $10 \mathrm{~K}$.

All images shown here were obtained after a sublimation time of $10 \mathrm{~min}$, resulting in coverage of approximately 0.4 monolayers. Subsequently, molecularly resolved images were taken in ultrahigh vacuum with an Omicron Nanotechnology (Taunusstein, Germany) variable-temperature atomic force microscope (AFM), operated in the frequency modulation noncontact (NC) mode. ${ }^{39}$

Molecules were deposited at a sample temperature of around $100 \mathrm{~K}$. The sample was transferred into the precooled NCAFM immediately after deposition and heated inside the NCAFM to the desired temperature. After the sample temperature had stabilized, the surface was imaged for at least $2 \mathrm{~h}$ with NCAFM. If only one phase was observed within these $2 \mathrm{~h}$, the phase is considered stable at that temperature. In case two phases were observed within the first $2 \mathrm{~h}$, the NC-AFM experiment was continued. If there were still two phases on the surface even after $24 \mathrm{~h}$ in total, the coexistence of these phases is considered stable at that temperature. In case the phase transition was fast enough to be completed within the first $24 \mathrm{~h}$, the remaining phase was observed for another $2 \mathrm{~h}$ to ensure no further phase transition starts. In this case, the remaining phase is considered stable at that temperature.

\section{ASSOCIATED CONTENT}

\section{S Supporting Information}

The Supporting Information is available free of charge on the ACS Publications website at DOI: 10.1021/acs.jpcc.6b05402.

Additional experimental details, Gibbs free energy calculations for the striped (Table S1, Figures S1 and S2) and the dense (Figures S3 and S4, Table S2) phases characterization, free energy calculations (Figure S5), Mulliken charge analysis (Figure S5), evaluation of the molecule-molecule and molecule-surface interaction 
energies, and nudge elastic band (NEB) calculation for the DHBA dimer dissociation (Figure S9) (PDF)

\section{AUTHOR INFORMATION}

\section{Corresponding Authors}

*E-mail chiara.1.paris@kcl.ac.uk.

*E-mail lev.kantorovitch@kcl.ac.uk.

*E-mail afloris@lincoln.ac.uk.

*E-mail kuehnle@uni-mainz.de.

\section{Notes}

The authors declare no competing financial interest.

\section{ACKNOWLEDGMENTS}

C.P. thanks the ACRITAS Marie Curie Initial Training Network (ITN) project for funding. Via our membership in the UK's HPC Materials Chemistry Consortium, which is funded by EPSRC (EP/F067496, EP/L000202), this work made use of the facilities of HECTOR and ARCHER, the UK's national high-performance computing service, which is funded by the Office of Science and Technology through EPSRC's High End Computing Programme. Financial support from the EU through grant PAMS (seventh framework program GA 610446) is gratefully acknowledged.

\section{REFERENCES}

(1) Lehn, J.-M. Supramolecular Chemistry-Molecules, Supermolecules, and Molecular Functional Units (Nobel lecture). Angew. Chem. Int. Ed. Engl. 1988, 27, 89-112.

(2) Barth, J. V. Molecular Architectonic on Metal Surfaces. Annu. Rev. Phvs. Chem. 2007, 58, 375-407.

(3) Rosei, F.; Schunack, M.; Naitoh, Y.; Jiang, P.; Gourdon, A.; Lægsgaard, E.; Stensgaard, I.; Joachim, C.; Besenbacher, F. Properties of Large Organic Molecules on Metal Surfaces. Prog. Surf. Sci. 2003, $71,95-146$

(4) Pawin, G.; Wong, K. L.; Kwon, K. Y.; Bartels, L. A Homomolecular Porous Network at a $\mathrm{Cu}(111)$ surface. Science 2006, 313, 961-962.

(5) Pawin, G.; Wong, K. L.; Kim, D.; Sun, D. Z.; Bartels, L.; Hong, S.; Rahman, T. S.; Carp, R.; Marsella, M. A Surface Coordination Network Based on Substrate-Derived Metal Adatoms with Local Charge Excess. Angew. Chem. Int. Ed. 2008, 47, 8442-8445.

(6) Phillips, A. G.; Perdigao, L. M. A.; Beton, P. H.; Champness, N. R. Tailoring Pores for Guest Entrapment in a Unimolecular Surface Self-Assembled Hydrogen Bonded Network. Chem. Commun. 2010, 46, 2775-2777.

(7) Böhringer, M.; Morgenstern, K.; Schneider, W.-D.; Berndt, R.; Mauri, F.; De Vita, A.; Car, R. Two-Dimensional Self-Assembly of Supramolecular Clusters and Chains. Phvs. Rev. Lett. 1999, 83, 324.

(8) Abdurakhmanova, N.; Floris, A.; Tseng, T. C.; Comisso, A.; Stepanow, S.; De Vita, A.; Kern, K. Stereoselectivity and Electrostatics in Charge-Transfer Mn- and Cs-TCNQ 4 Networks on $\mathrm{Ag}(100)$. Nat. Commun. 2012, 3, 940.

(9) Floris, A.; Comisso, A.; De Vita, A. Fine-Tuning the Electrostatic Properties of an Alkali-Linked Organic Adlayer on a Metal Substrate. ACS Nano 2013, 7, 8059-8065.

(10) Della Pia, A.; Riello, M.; Floris, A.; Stassen, D.; Bonifazi, D.; Jones, T. S.; De Vita, A.; Costantini, G. Anomalous Coarsening Driven by Reversible Charge Transfer at Metal-Organic Interfaces. ACS Nano 2014, 8, 12356-12364.

(11) Willner, A. E.; Byer, R. L.; Chang-Hasnain, C. J.; Forrest, S. R.; Kressel, H.; Kogelnik, H.; Tearney, G. J.; Townes, C. H.; Zervas, M. N. Optics and Photonics: Key Enabling Technologies. Proc. IEEE 2012, 100, 1604-1643.

(12) Dimitrakopoulos, C. D.; Purushothaman, S.; Kymissis, J.; Callegari, A.; Shaw, J. W. Low-Voltage Organic Transistors on Plastic
Comprising High-Dielectric Constant Gate Insulators. Science 1999, 283, 822-824.

(13) Joachim, C.; Gimzewski, G. K.; Aviram, A. Electronics Using Hybrid-Molecular and Mono-Molecular Devices. Nature 2000, 408, 541-548.

(14) Nilius, N.; Risse, T.; Schauermann, S.; Shaikhutdinov, S.; Sterrer, M.; Freund, H.-J. Model Studies in Catalysis. Top. Catal. 2011, $54,4-12$.

(15) Rahe, P.; Kittelmann, M.; Neff, J. L.; Nimmrich, M.; Reichling, M.; Maass, P.; Kühnle, A. Tuning Molecular Self-Assembly on Bulk Insulator Surfaces by Anchoring of the Organic Building Blocks. $\underline{A d v}$. Mater. 2013, 25, 3948-3956.

(16) Pawlak, R.; Nony, L.; Bocquet, F.; Oison, V.; Sassi, M.; Debierre, J. M.; Loppacher, C.; Porte, L. Supramolecular Assemblies of 1,4-Benzene Diboronic Acid on $\mathrm{KCl}(001)$. I. Phvs. Chem. C 2010, 114, 9290-9295.

(17) Rahe, P.; Lindner, R.; Kittelmann, M.; Nimmrich, M.; Kühnle, A. From Dewetting to Wetting Molecular Layers: $\mathrm{C}_{60}$ on $\mathrm{CaCO}_{3}(10 \overline{1} 4)$ as a Case Study. Phvs. Chem. Chem. Phys. 2012, 14, 6544-6548.

(18) Kunstmann, T.; Schlarb, A.; Fendrich, M.; Wagner, T.; Möller, R; Hoffmann, R. Dynamic Force Microscopy Study of 3,4,9,10Perylenetetracarboxylic Dianhydride on $\mathrm{KBr}(001)$. Phvs. Rev. B: Condens. Matter Mater. Phys. 2005, 71, 121403.

(19) Nony, L.; Bennewitz, R.; Pfeiffer, O.; Gnecco, E.; Baratoff, A.; Meyer, E.; Eguchi, T.; Gourdon, A.; Joachim, C. Cu-TBPP and PTCDA Molecules on Insulating Surfaces Studied by Ultra-HighVacuum Non-Contact AFM. Nanotechnology 2004, 15, S91.

(20) Hauke, C. M.; Bechstein, R.; Kittelmann, M.; Storz, C.; Kilbinger, A. F.; Rahe, P.; Kühnle, A. Controlling Molecular SelfAssembly on an Insulating Surface by Rationally Designing an Efficient Anchor Functionality That Maintains Structural Flexibility. ACS Nano 2013, 7, 5491.

(21) Rahe, P.; Nimmrich, M.; Greuling, A.; Schütte, J.; Stará, I. G.; Rybáček, J.; Huerta-Angeles, G.; Starý, I.; Rohlfing, M.; Kühnle, A. Toward Molecular Nanowires Self-Assembled on an Insulating Substrate: Heptahelicene-2-carboxylic Acid on Calcite (1014). I. Phys. Chem. C 2010, 114, 1547-1552.

(22) Kittelmann, M.; Rahe, P.; Kühnle, A. Molecular Self-Assembly on an Insulating Surface: Interplay Between Substrate Templating and Intermolecular Interactions. I. Phvs.: Condens. Matter 2012, 24, 354007.

(23) Neff, J. L.; Söngen, H.; Bechstein, R.; Maass, P.; Kühnle, A. Long Range Order Induced by Intrinsic Repulsion on an Insulating Substrate. I. Phvs. Chem. C 2015, 119, 24927-24931.

(24) Kittelmann, M.; Nimmrich, M.; Neff, J. L.; Rahe, P.; Greń, W.; Bouju, X.; Gourdon, A.; Kühnle, A. Controlled Activation of Substrate Templating in Molecular Self-Assembly by Deprotonation. I. Phvs. Chem. C 2013, 117, 23868-23874.

(25) Kittelmann, M.; Rahe, P.; Gourdon, A.; Kühnle, A. Direct Visualization of Molecule Deprotonation on an Insulating Surface. ACS Nano 2012, 6, 7406-7411.

(26) Adam, M. S.; Gutmann, M. J.; Leech, C. K.; Middlemiss, D. S.; Parkin, A.; Thomas, L. H.; Wilson, C. C. Stability and Cooperativity of Hydrogen Bonds in Dihydroxybenzoic Acids. New I. Chem. 2010, 34, $85-91$.

(27) Mulliken, R. S. Electronic Population Analysis on LCAO-MO Molecular Wave Functions. I. Chem. Phvs. 1955, 23, 1833-1840.

(28) Neff, J. L.; Rahe, P. Insights Into Kelvin Probe Force Microscopy Data of Insulator-Supported Molecules. Phvs. Rev. B: Condens. Matter Mater. Phys. 2015, 91, 085424-085435.

(29) Jorgensen, W. L.; Severance, D. L. Aromatic-Aromatic Interactions: Free Energy Profiles for the Benzene Dimer in Water, Chloroform, and Liquid Benzene. I. Am. Chem. Soc. 1990, 112, 47684774.

(30) VandeVondele, J.; Krack, M.; Mohamed, F.; Parrinello, M.; Chassaing, T.; Hutter, J. Quickstep: Fast and Accurate Density Functional Calculations Using a Mixed Gaussian and Plane Waves Approach. Comput. Phys. Commun. 2005, 167, 103-128. 
(31) URL http://www.cp2k.org/.

(32) Goedecker, S.; Teter, M.; Hutter, J. Separable Dual-Space Gaussian Pseudopotentials. Phvs. Rev. B: Condens. Matter Mater. Phvs. 1996, 54, 1703-1710.

(33) Perdew, J. P.; Burke, K.; Ernzerhof, M. Generalized Gradient Approximation Made Simple. Phvs. Rev. Lett. 1996, 77, 3865-3868.

(34) Grimme, S. Semiempirical GGA-Type Density Functional Constructed With a Long-Range Dispersion Correction. I. Comput. Chem. 2006, 27, 1787-1799.

(35) Giannozzi, P. et al. QUANTUM ESPRESSO: a Modular and Open-Source Software Project for Quantum Simulations of Materials, I. Phvs.: Condens. Matter 2009, 21, 395502-395521; URL http:// www.quantum-espresso.org.10.1088/0953-8984/21/39/395502.

(36) URL http://www.mth.kcl.ac.uk/ lev/codes/lev00/index.html.

(37) Kantorovich, L. Quantum Theory of the Solid State: An Introduction: Kluwer: London, 2004.

(38) Jónsson, H.; Mills, G.; Jacobsen, K. W. Nudged Elastic Band Method for Finding Minimum Energy Paths of Transitions. In Classical and Quantum Dynamics in Condensed Phase Simulations; Berne, B. J., Ciccotti, G., Coker, D. F., Eds.; World Scientific: 1998; p 385.

(39) Albrecht, T. R.; Grütter, P.; Horne, D.; Rugar, D. Frequency Modulation Detection Using High-Q Cantilevers for Enhanced Force Microscope Sensitivity. L. Appl. Phys. 1991, 69, 668-673. 